participant per arm died, both unrelated to intervention. Qualitative data suggests feasibility and acceptability.

A randomised double-blind phase III trial of mirtazapine is feasible and acceptable in patients with COPD/ILD, with low toxicity, and is warranted.

\section{NURSING STAFF ATTITUDES TO CLINICALLY ASSISTED HYDRATION AT THE END OF LIFE IN A HOSPICE SETTING}

Stephen Cox, Charlotte Brigden, Ruth Harris-Skillman, Thomas Watkins. Pilgrim Hospices in East Kent, UK

10.1136/spcare-2019-mariecuriepalliativecare.34

Background Clinically assisted hydration (CAH) at end of life is a contentious issue (Cabañero-Martinez et al, 2016) There is disagreement as to its benefits and this can lead to a lack of concordance between medical and nursing opinions, with nurses feeling unable to challenge doctors (Churchman, 2010; Van Der Riet, 2008; Mayashita, 2007). Prior to introducing a feasibility study on CAH (Davies, 2018) on one hospice site, an unpublished survey of nursing staff revealed widespread dissatisfaction with the standard practice of not routinely offering CAH. Following completion of the study a staff survey was undertaken to see if attitudes had changed.

Aims To determine if nurses feel coerced into accepting the current hospice policy on CAH. To examine how nurses interacted with families when asked about CAH. To explore staff engagement with research on $\mathrm{CAH}$.

Method A semi-structured questionnaire sent to all nursing staff including HCA's, with closed and open-ended questions. The responses were analysed using descriptive statistics and thematic analysis.

Results The responses indicated that;

The majority accept standard care without feeling coerced $(68 \%)$.

Nurses felt they could challenge accepted practice (62\%).

An overwhelming majority felt the hospice should be engaged in research on $\mathrm{CAH}(80 \%)$.

Nursing staff expressed the following;

Nurses wanted to be more involved in decision making around $\mathrm{CAH}$.

Many felt that a standard practice should be replaced with individual patient decisions. Staff were frequently asked about $\mathrm{CAH}$ and felt able to discuss the risks and benefits with families.

Conclusions Overall, nurses accepted hospice standard practice on $\mathrm{CAH}$ and did not feel coerced. A few opposed further research, most welcomed it to establish an evidence base.

\section{PAIN IN ADVANCED DISEASE: INITIAL FINDINGS FROM A QUALITATIVE INTERVIEW STUDY WITH BRITISH SOUTH ASIANS IN LEEDS AND BRADFORD, UK}

\footnotetext{
1,2Gemma Clarke, 'Shenaz Ahmed, ${ }^{3}$ Wali Nazar, ${ }^{2}$ Clare Rayment, ${ }^{1}$ Michael Bennett. ${ }^{1}$ University of Leeds, UK; ${ }^{2}$ Marie Curie Hospice Bradford, UK; ${ }^{3}$ Bradford Community Specialist Palliative Care Team, UK
}

\subsection{6/spcare-2019-mariecuriepalliativecare.35}

Background Population projections indicate that by 2051, the Black, Asian and Minority Ethnic (BAME) populations of England and Wales will rise to 3.8 million people aged 65 years and over, and 2.8 million aged 70 and over (Lievesley, 2010). This has important implications for palliative care services. Current evidence on ethnicity and palliative care is limited, and is often solely focused on access to services (Calanzani et al., 2013). Evidence is particularly limited for British South Asian populations (Shabnam, et al, 2019). South Asians have a high burden of life-limiting and chronic diseases compared to other groups (Nair and Prabhakaran, 2012). This study focuses on pain management, the impact of culture, and the quality of services for British South Asians with advanced disease.

Aim To explore the attitudes and perceptions of British South Asians with advanced disease concerning pain, pain management, and expression of pain; and to examine the differences in service provision.

Methods Qualitative interviews $(\mathrm{N}=25)$ with individuals who have serious life-limiting disease, are experiencing pain as a symptom, or having their pain controlled, and are from a South Asian background currently living in Leeds or Bradford UK. Fieldwork was undertaken in 2019. Interviews were audio recorded, transcribed verbatim and analysed using NVivo and Thematic Analysis (Braun and Clarke, 2006).

Discussion Key themes: experiences of pain and pain management related to cultural background; help-seeking behaviour and barriers; strong pain management at the end of life; and differences between those utilising specialist palliative care services and those receiving care elsewhere.

\section{PREFERRED AND ACTUAL PLACE OF DEATH IN PATIENTS WITH HAEMATOLOGICAL MALIGNANCIES}

${ }^{1}$ Rebecca Sheridan, ${ }^{1}$ Eve Roman, ${ }^{1}$ Alexandra Smith, ${ }^{2}$ Russell Patmore, ${ }^{3}$ Martin Howard, ${ }^{1}$ Debra Howell. ${ }^{1}$ Epidemiology and Cancer Statistics Group, Department of Health Sciences, University of York, UK; ${ }^{2}$ Queen's Centre for Oncology and Haematology, Castle Hill Hospital, UK; ${ }^{3}$ Department of Haematology, York Hospital, UK

\subsection{6/spcare-2019-mariecuriepalliativecare.36}

Patients with haematological malignancies are more likely to die in hospital than those with other cancers (Cohen et al. 2015), but little is known about their preferred place of death and the discussions surrounding this. This study aimed to investigate this using data from the Haematological Malignancy Research Network (www.HMRN.org); a population-based cohort that, since 2004, has collected detailed information on all newly diagnosed patients across 14 hospitals (catchment populations $\sim 4$ million). Additional data for the present study were collected from the medical records of 892 HMRN patients (>18 years) who died 2011-2012.

Fifty-eight percent of patients died in hospital (home, 20\%; care home, 11.9\%; hospice, 10.2\%). Half had a preferred place discussion recorded, with the majority of these having more than one discussion. At the discussion nearest death, the most common preferred place was home (44.9\%)followed by hospice (20\%), hospital (19.5\%), and care home (15.6\%). However, preferences varied over time; for patients whose final recorded preference was hospital, $14.3 \%$ had the same preference at their first discussion. When home was the final choice, $80.4 \%$ also chose home at their first discussion. Patient involvement was less common during final discussions. Almost all of those who wanted to die in hospital did so, yet only half of those who wanted to die at home achieved this. 